\title{
Evidence for the Large-Scale Dissociation of Molecular Gas in the Inner Spiral Arms of M81
}

\author{
R. J. Allen \\ Space Telescope Science Institute, 3700 San Martin Drive \\ Baltimore, MD 21218, USA (rjallen@stsci.edu) \\ J. H. Knapen \\ Department of Physical Sciences, University of Hertfordshire \\ College Lane, Hatfield, Herts AL10 9AB, UK (knapen@star.herts.ac.uk) \\ R. Bohlin \\ Space Telescope Science Institute, 3700 San Martin Drive \\ Baltimore, MD 21218, USA (bohlin@stsci.edu) \\ and \\ T. P. Stecher \\ Code 680, Goddard Space Flight Center \\ Greenbelt, MD20771 (stecher@uit.gsfc.nasa.gov)
}

\begin{abstract}
We compare the detailed distributions of $\mathrm{HI}, \mathrm{H} \alpha$, and $150 \mathrm{~nm}$ far-UV continuum emission in the spiral arms of M81 at a resolution of $9^{\prime \prime}$ (linear resolution $150 \mathrm{pc}$ at $3.7 \mathrm{Mpc}$ distance). The bright $\mathrm{H} \alpha$ emission peaks are always associated with peaks in the far-UV emission. The converse is not always true; there are many regions of farUV emission with little corresponding $\mathrm{H} \alpha$. The $\mathrm{HI}$ and the far-UV are always closely associated, in the sense that the $\mathrm{HI}$ is often brightest around the edges of the far-UV emission.

The effects of extinction on the morphology are small, even in the far-UV. Extensive far-UV emission, often with little corresponding $\mathrm{H} \alpha$, indicates the presence of many "Bstars", which produce mostly non-ionizing UV photons. These far-UV photons dissociate a small fraction of an extensive layer of $\mathrm{H}_{2}$ into $\mathrm{H} \mathrm{I}$.

The observed morphology can be understood if "chimneys" are common in the spiral arms of M81, where holes are blown out of the galactic disk, exposing the bright $\mathrm{H}$ II regions and the corresponding far-UV associated with vigorous star formation. These "naked" star-forming regions show little obscuration. $\mathrm{H}_{2}$ is turned into $\mathrm{H}$ I by UV photons impinging on the interior surfaces of these chimneys.
\end{abstract}


The intensity of the far-UV radiation measured by UIT can dissociate the underlying $\mathrm{H}_{2}$ with a typical density of $\sim 10 \mathrm{H}$ nucleii $\mathrm{cm}^{-3}$ to produce the observed amount of $\mathrm{H}$ I in the spiral arms of M81. Except for thin surface layers locally heated in these photo-dissociation regions close to the far-UV sources, the bulk of the molecular gas in the inner disk of M81 is apparently too cold to produce much ${ }^{12} \mathrm{CO}(1-0)$ emission.

Subject headings: galaxies: individual: M81 - galaxies: ISM - ISM: molecules - ISM: clouds - radio lines: galaxies

Accepted for publication in the Astrophysical Journal 


\section{Introduction}

The possibility that an appreciable fraction of the atomic hydrogen (H I) found in the spiral arms of disk galaxies is dissociated molecular gas was first suggested about a decade ago from a comparison of the relative placement of the dust lanes, $\mathrm{H}$ II regions $(\mathrm{H} \alpha)$, and $\mathrm{HI}$ ridges in parts of M83 (Allen, Atherton, \& Tilanus 1985, 1986) and M51 (Tilanus \& Allen 1987). This interpretation of the observations is based on the density-wave model for spiral structure, in which the temporal sequence of massive star formation is spread out in space across a spiral arm by the action of a density wave driving the interstellar gas. More detailed work has subsequently been done, including comparisons with other spiral tracers, such as the nonthermal radio continuum and the $\mathrm{CO}$ emission, for M83 (Tilanus \& Allen 1993), for M51 (Vogel, Kulkarni, \& Scoville 1988; Tilanus \& Allen 1989, 1990, 1991; Rand, Kulkarni, \& Rice 1992; Knapen et al. 1992), and more recently for M100 (Knapen \& Beckman 1994, 1996).

Shaya \& Federman (1987) provided the first theoretical discussion of the UV photodissociation process as an explanation for the generally-flat radial distribution of $\mathrm{H}$ I in galaxies. More recently, from observations of the [C II] emission from galaxies and quantitative modelling of the results, Stacey et al. (1991) have confirmed that photodissociation regions (PDRs; e.g. Hollenbach \& Tielens 1995) are the sites of substantial H I production in galaxy disks. Madden et al. (1993) have even suggested that, in their preferred model, virtually all of the $\mathrm{H}$ I in the disk of NGC 6946 could be produced in PDRs. Unfortunately, the angular resolution available with current instrumentation in the [C II] line at $158 \mu$ is inadequate for the study of individual PDRs in nearby galaxies.

The morphological details of tracers in spiral arms represent new evidence for the production of $\mathrm{HI}$ by the extensive dissociation of $\mathrm{H}_{2}$ in PDRs. There are two new elements in the present study. First, M81 (NGC3031) is a galaxy which is not bright in CO emission (e.g. Brouillet et al. 1991). The conventional interpretation using $\mathrm{CO}$ as a tracer for $\mathrm{H}_{2}$ is that this galaxy contains relatively little molecular gas, contrary to the situation for the previously-studied galaxies M83, M51, and M100. Second, the availability of UV image data for M81 from the Ultraviolet Imaging Telescope (UIT) permits a comparison of the H I morphology to the distribution of UV photons at $\lambda \approx 150$ $\mathrm{nm}(\approx 8.3 \mathrm{eV})$, in the middle of the range of photon energies which are important for the dissociation of $\mathrm{H}_{2}$ (Stecher \& Williams 1967) . To interpret the UV data, any effects of patchy obscuration by dust must be understood to properly compare the UV and $\mathrm{H}$ I morphologies. $\mathrm{H} \alpha$ images of M81 aid in our investigation of this effect. In our Galaxy, the far-UV extinction (in mags) at $\lambda \approx 150 \mathrm{~nm}$ is greater than the extinction at $\mathrm{H} \alpha(\lambda \approx 650 \mathrm{~nm})$ by a factor of more than 3 . Therefore, any effect of varying obscuration on the morphology of the $\mathrm{H} \alpha$ in M 81 is greatly magnified in the far UV.

\section{Observations}

The 640 sec far-UV exposure of M81 (FUV0556; Hill et al. 1995) is from the UIT/ASTRO-1 archive, and is centered at $\lambda=152 \mathrm{~nm}$ with bandwidth 35.4 $\mathrm{nm}$ (Stecher et al. 1992). The VLA H I data were taken by B. Hine \& A. Rots, and are provided by D. Westpfahl[2]. We used the H I data cube to calculate a map of $\mathrm{H}$ I column density $\mathrm{N}(\mathrm{HI})$. Only those pixels with absolute values in excess of 2.5 times the rms noise in the channel maps are used in the moment calculation, with the additional restriction that such values must occur in at least two adjacent velocity channels (which are separated by $10 \mathrm{~km} \mathrm{~s}^{-1}$ ). M. Kaufman and N. Devereux provided $\mathrm{H} \alpha$ images of M81. Although we use the newer data (Devereux, Jacoby, \& Ciardullo 1995) for our illustrations, the older data (Kaufman et al. 1989a) provide an important "second opinion" in a few cases where smoothed cosmic-ray hits on the CCD look like H II regions, or where foreground stars are imperfectly subtracted.

In combining the different data sets, we first ensure that the astrometry of the individual images is consistent by checking the positions of the center of the galaxy and several foreground stars (UV and $\mathrm{H} \alpha$ )

\footnotetext{
${ }^{1}$ Photons with energies in the range 11.2 to $13.6 \mathrm{eV}$ are required to start the process, by pumping the $\mathrm{H}_{2}$ molecules into higher vibrational states of the ground electronic state (through Lyman-Werner band transitions). Subsequently, photons with lower energies down to $\sim 6.6 \mathrm{eV}$ can then also dissociate $\mathrm{H}_{2}$ by facilitating transitions into the vibrational continuum of the ground electronic state.

${ }^{2}$ New VLA H I data on M81 have recently been published by Adler \& Westpfahl (1996). The sensitivity of the new data is significantly better than that of our older VLA data. However, we are primarily interested in the bright features on the $\mathrm{HI}$ image, and these are not noise limited in the older data. Furthermore, the spatial resolution of our data is better ( $9^{\prime \prime} \mathrm{vs}$. $12^{\prime \prime}$ ) and more suitable for comparison with the UV image.
} 
and the location and orientation of the general spiral arm features (H I). The original astrometry provided with the data sets is excellent. Any difference in alignment between images appears to be no greater than $1^{\prime \prime}-2^{\prime \prime}$, which is negligible in comparison with the final resolution of $9^{\prime \prime}$ used here.

The next step is to smooth the different images to a common resolution of $9^{\prime \prime}$, which is that of the $\mathrm{HI}$ image (linear resolution 150 pc at our adopted distance of $3.7 \mathrm{Mpc}$ ). The angular resolutions of the UV and $\mathrm{H} \alpha$ images are determined by fitting 2D Gaussian profiles to a number of point-like sources in the frames. We then convolve the images with the appropriate Gaussians in order to obtain smoothed images at the resolution of the $\mathrm{HI}$ image. The images are then interpolated to a common pixel grid in order to facilitate a direct comparison of the positions of various features.

\section{Results and Analysis}

Full-sized UIT images of M81 in the near- and farUV (FUV) are shown as Figures $1 \& 2$ in Hill et al. (1995) and are not duplicated here. Similarly, the full $\mathrm{H} \alpha$ image is Figure 1 in Devereux et al. (1995). Our independent derivation of the $\mathrm{H}$ I column density map is essentially identical to Figure 13 in Kaufman et al. (1989a). For reference, Figure 11 shows a contour representation of the FUV image (smoothed to $9^{\prime \prime}$ ), overlaid on a grey-scale representation of the $\mathrm{H}$ I image of the galaxy. Five frames indicate the locations of the sub-fields, which we will discuss in more detail below. First, the radial distributions of the three tracers are considered.

\subsection{Radial distributions}

Figure 2 shows the radial distributions of the three tracers: H I (bottom panel), FUV (middle panel), and $\mathrm{H} \alpha$ (top panel). The curves are derived from the input images by averaging in elliptical annuli of widths $20^{\prime \prime}$ spaced every $20^{\prime \prime}$ ( $\approx 330 \mathrm{pc}$ ) along the major axis. We used $i=59^{\circ}$ for the inclination angle of the galaxy, and $\mathrm{PA}=157^{\circ}$ for the position angle of the major axis (de Vaucouleurs et al. 1991); these values were kept constant for all ellipses and for each input image data set.

The large-scale axially-symmetric morphology of the galaxy is reproduced clearly in the profiles of Fig.2. Both the FUV and $\mathrm{H} \alpha$ are centrally peaked, whereas the $\mathrm{H}$ I profile reflects the central deficiency of atomic hydrogen seen in Fig.11. From $3^{\prime}$ to $11^{\prime}$ the three tracers show similar features: First, the FUV and $\mathrm{H} \alpha$, which trace massive star formation, both rise to their maximum values near $R \approx 5^{\prime}$, after which they both generally decline. More surprising is the detailed correlation of the peaks and valleys of these two profiles between $5^{\prime}$ and $10^{\prime}$ radius, which strongly suggests that dust extinction does not appreciably affect the morphology of either the FUV or the $\mathrm{H} \alpha$ emission. In the following section we show that even the details of the FUV and $\mathrm{H} \alpha$ images on the $\approx 150 \mathrm{pc}$ scale appear to be largely unaffected by dust. Second, the $\mathrm{H}$ I distribution shows roughly the same behavior as the massive-star tracers. The steep rise between $3^{\prime}$ and $5^{\prime}$ discussed above is clearly reproduced in $\mathrm{HI}$, as are the relative dip around $R \approx 7^{\prime}$ and the secondary peak at $R \approx 8.5^{\prime}$. The conventional interpretation of this behavior is simply that more $\mathrm{HI}$ leads to more star formation. However, our preferred alternative interpretation is that the $\mathrm{HI}$ radial profile naturally follows those of FUV and $\mathrm{H} \alpha$ because the $\mathrm{HI}$ is a product of the star formation, through dissociation, rather than a precursor to it. Thus, where the star formation is enhanced, more $\mathrm{H} \mathrm{I}$ is produced, which is just what the radial profiles show.

\subsection{Detailed morphology}

Earlier work on M81 (e.g. Kaufman et al. 1989a) and on many other nearby galaxies shows that the $\mathrm{H}$ I and the $\mathrm{H} \alpha$ trace spiral structure in much the same general way at a linear resolution of $\sim 1 \mathrm{kpc}$. Hill et al. (1995) also showed the excellent correspondence between $\mathrm{H} \alpha$ peaks and FUV peaks in M81. The H IFUV images overlaid in Fig 1 further illustrate this general coincidence. However the detailed correspondence between FUV, $\mathrm{H} \alpha$, and $\mathrm{HI}$ emission is more complicated. To illustrate this richness of detail, we present and discuss the images in 5 fields positioned as indicated in Fig.1. In Figs. 37, we show for each field first the contours of the FUV emission overlaid on a grey-scale representation of itself, then overlaid on the $\mathrm{H} \alpha$, and finally on the $\mathrm{H}$ I emission. In each middle panel we also mark the $B$-band dust filaments of Kaufman, Elmegreen \& Bash (1989b).

\subsubsection{Far $U V-H \alpha$ comparison:}

Dust in the Galaxy is unevenly distributed, and the continuum extinction at $\lambda \approx 150 \mathrm{~nm}$ is about 4 times that at visual wavelengths. Therefore the FUV image ought to show significantly more small-scale structure 
than the $\mathrm{H} \alpha$ image. Instead, the opposite occurs; the FUV emission is smoother and spread more widely than the $\mathrm{H} \alpha$, as can be seen from a comparison of the "FUV-on-FUV" and "FUV-on-H $\alpha$ " panels in Figs. 曻 8.

There are two aspects to this unexpected $\mathrm{H} \alpha$-FUV correspondence. First, every reliable bright peak in the $\mathrm{H} \alpha$ has a counterpart in the FUV. Second, there are many places with extended and relatively intense FUV emission which have little or no associated $\mathrm{H} \alpha$.

The fact that every reliable bright peak in the $\mathrm{H} \alpha$ has a counterpart in the FUV must mean that the effects of extinction on the morphology are small on the scale of $150 \mathrm{pc}$, so that either both the $\mathrm{H} \alpha$ and the FUV appear either essentially unobscured, or both are partly hidden in approximately the same way. The extinction may also be so high that neither the FUV nor the $\mathrm{H} \alpha$ are visible ${ }^{3}$.

There are a few regions which appear to emit relatively strong $\mathrm{H} \alpha$ but do not have a counterpart in FUV. Such regions are for instance located at $09^{\mathrm{h}} 52^{\mathrm{m}} 12^{\mathrm{s}}, 69^{\circ} 18^{\prime} 15^{\prime \prime}$ (in the middle of a UV void in Field 1), at $09^{\mathrm{h}} 50^{\mathrm{m}} 33^{\mathrm{s}}, 69^{\circ} 17^{\prime} 30^{\prime \prime}$ (linear structure $\sim 30^{\prime \prime}$ long in Field 3), or at $09^{\mathrm{h}} 50^{\mathrm{m}} 36^{\mathrm{s}}, 69^{\circ} 20^{\prime} 10^{\prime \prime}$ (small crescent-shaped object in Field 3). However, none of these $\mathrm{H} \alpha$ features appear on the older $\mathrm{H} \alpha$ image of Kaufman et al. (1989a) (not shown here), confirming that these features on the Devereux et al. $\mathrm{H} \alpha$ image are most probably not $\mathrm{H}$ II regions at all, but artifacts such as (smoothed) cosmic ray events or improperly-subtracted foreground stars 7 .

While every reliable bright peak on the $\mathrm{H} \alpha$ image has a counterpart in the FUV image with the same position and general shape, there are many places with extended and relatively intense FUV emission which have little or no associated $\mathrm{H} \alpha$. There are even regions of relatively bright FUV emission, such as those at $09^{\mathrm{h}} 52^{\mathrm{m}} 10^{\mathrm{s}}, 69^{\circ} 16^{\prime} 30^{\prime \prime}$ (Field 1 ), and $09^{\mathrm{h}} 51^{\mathrm{m}} 03^{\mathrm{s}}$, $69^{\circ} 23^{\prime} 30^{\prime \prime}$ (Field 4 ), which show hardly any counter-

\footnotetext{
${ }^{3}$ The regions of highest dust extinction appear to be filamentary, as indicated for example on the central panel of Figs. 3 . 7 in this paper. With such a patchy geometry it is unlikely that all parts of a large $\mathrm{H}$ II region consisting of half a dozen $\mathrm{O}$ stars could be hidden, as is corroborated by detailed comparisons of the thermal radio emission with the $\mathrm{H} \alpha$ in e.g. M101 (Israel, Goss, \& Allen 1975), NGC 6946 (van der Kruit, Allen, \& Rots 1977), and M51 (Tilanus et al. 1988). None of those galaxies appear to harbor large $\mathrm{H}$ II regions which are entirely hidden by dust.

${ }^{4}$ The older $\mathrm{H} \alpha$ image of Kaufman et al. also shows a number of "H II regions" which do not appear on the Devereux et al. Ho image.
}

parts in $\mathrm{H} \alpha$ at the present levels of sensitivity.

In principle, some of these UV sources could be artifacts on the UIT FUV image. There are five kinds of artifacts in the UIT data which could be important for us: 1) "Bright Spots": There are one or two bright spots in each UIT camera; they occur within 200 pixels of the edge of the image. The M81 image is in the central parts of the field, so we are not concerned with these artifacts. 2) Cosmic Rays: These are rare on UIT images, ranging from none to several per image. They are always very bright, saturated, and extended blobs which do not look like stars. The full-resolution UIT FUV frame we have used does not appear to have such features. 3) Foreground Galactic Stars: Typically there are one or two UV-bright Galactic foreground stars in each UIT field at the intermediate Galactic latitudes of M81. These are mostly $\mathrm{V} \approx 11 \mathrm{mag}$ A stars, so they are bright in optical CCD images. Again, there are no such stars known within the optical image of M81. 4) Scratches: The UIT detector is photographic film, and scratches can appear on it from the handling and developing processes. These are visible at faint levels, but are easily recognizable as long narrow parallel lines. Smoothing will reduce their impact, and our M81 image does not show the effects of scratches. 5) Dust Specks: During densitometry of the flight film in a clean room environment, some dust contamination still appears, which mimics stars. Since only the larger specks are important for this paper, the reality of all bright sources is easily confirmed by comparison with the UIT $128 \mathrm{sec}$ shorter exposure of the same field (FUV0557).

The features in M81 which are relatively strong in the UV but weak in $\mathrm{H} \alpha$ are not artifacts. Apparently, the stars which power these regions produce ample amounts of dissociating FUV photons at $\lambda \approx 150 \mathrm{~nm}$, but relatively few ionizing photons at $\lambda \leq 91.2 \mathrm{~nm}$. We shall refer here to this stellar population as "Bstars", but recognize that late $\mathrm{O}$ and early A stars may be included. Differences in the relative amounts of $\mathrm{UV}$ and $\mathrm{H} \alpha$ in any given region could arise from differences in age, as has been discussed for M81 by Hill et al. (1995), and/or from variations in the IMF.

\subsubsection{Far $U V-H$ I comparison:}

From the third panel in Figs.357, $\mathrm{HI}$ is often found alone at faint levels in the spiral arms, without closely-associated UV and with little or no $\mathrm{H} \alpha$ at the present sensitivity. However, in the neighborhood of a FUV concentration, the Hi tends to be 
brighter, and the brightest peaks are located at the periphery of the UV concentrations rather than at the position where the UV has its local maximum. The H I may show a peak slightly offset from the FUV peak, for example in the small UV region near $09^{\mathrm{h}} 52^{\mathrm{m}} 02^{\mathrm{s}}, 69^{\circ} 19^{\prime} 30^{\prime \prime}$ (Field 1); but, more often, the $\mathrm{HI}$ forms an elongated patch next to the FUV, such as near $09^{\mathrm{h}} 52^{\mathrm{m}} 19^{\mathrm{s}}, 69^{\circ} 12^{\prime} 30^{\prime \prime}$ (Field 2), or $09^{\mathrm{h}} 50^{\mathrm{m}} 31^{\mathrm{s}}$, $69^{\circ} 20^{\prime} 45^{\prime \prime}$ (Field 3). On a larger scale, H I ridges a kiloparsec $\left(\sim 1^{\prime}\right)$ or more in length are found preferentially alongside UV ridges, such as the U-shaped $\mathrm{H}$ I feature stretching across Field 2 along the northern edge of the UV near $09^{\mathrm{h}} 52^{\mathrm{m}} 00^{\mathrm{s}}, 69^{\circ} 13^{\prime} 20^{\prime \prime}$, and the $\mathrm{H}$ I ridge stretching in a N-S direction just to the east of the UV in Field 3 near $09^{\mathrm{h}} 50^{\mathrm{m}} 40^{\mathrm{s}}, 69^{\circ} 19^{\prime} 00^{\prime \prime}$.

Some of the most interesting cases are those where the HI surrounds the UV concentration, such as the FUV peak sitting inside the $\mathrm{H}$ I depression near $09^{\mathrm{h}} 50^{\mathrm{m}} 39^{\mathrm{s}}, 69^{\circ} 18^{\prime} 45^{\prime \prime}$ (Field 3), and another at $09^{\mathrm{h}} 52^{\mathrm{m}} 14^{\mathrm{s}}, 69^{\circ} 18^{\prime} 40^{\prime \prime}$ (Field 1). A rather spectacular example of this morphology is the region near $9^{\mathrm{h}} 50^{\mathrm{m}} 44^{\mathrm{s}}, 69^{\circ} 17^{\prime} 30^{\prime \prime}$ (Field 3) where the H I forms a "halo" surrounding two peaks of a large UV-emitting region. The northern peak of this pair corresponds with the relatively bright $\mathrm{H}$ II region \#44 in the cata$\log$ of Petit, Sivan, \& Karachentsev (1988) and is also \#131 in Kaufman et al. (1987). The southern peak is actually a loose group of small, faint $\mathrm{H}$ II regions including \#55 in Petit et al. and several others, which also appear in Fig.3 of Kaufman et al. (1987) but are apparently too faint to be catalogued there.

\subsection{Statistics of the associations}

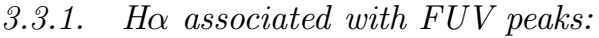

In the five fields we have identified a total of 144 sites of locally-enhanced FUV emission ("FUV peaks"), of which 21 are "strong"

$\left(\geq 10 \times 10^{-18} \mathrm{erg} \mathrm{cm}^{-2} \mathrm{~s}^{-1} \AA^{-1} \operatorname{arcsec}^{-2}\right)$. In $63 \%$ of the cases (90), $\mathrm{H} \alpha$ emission is associated and coincident with the FUV peak, in $13 \%$ (18) the associated $\mathrm{H} \alpha$ emission is slightly offset. For $10 \%$ (14 peaks) of the FUV peaks no associated $\mathrm{H} \alpha$ emission is evident on our images at the present level of sensitivity. Another 15\% (22) could not be easily classified; this category includes regions with diffuse $\mathrm{H} \alpha$ emission, possible unsubtracted residuals from foreground stars in the $\mathrm{H} \alpha$ image, and sources on the UV image which may be Galactic foreground stars.

\subsubsection{HI associated with FUV peaks:}

Of the 144 FUV peaks, only $8 \%$ (11 peaks) do not show any appreciable $\mathrm{HI}$ emission that can reasonably be considered as associated with the UV peak (i.e. within $\sim 200 \mathrm{pc}$ ). In $11 \%$ of the cases (16) the $\mathrm{HI}$ is coincident with the FUV, but in a striking majority of $81 \%$ of all FUV peaks the H I is seen offset from the FUV. The latter category includes cases where the bright $\mathrm{H}$ I peak is offset from the FUV, but there is still emission from the wings of the $\mathrm{HI}$ extending towards the position of the FUV peak. Of our 21 strong FUV peaks, one (5\%) may have coincident $\mathrm{H}$ I emission, but 20, or possibly all (100\%) have associated $\mathrm{H}$ I emission offset from the FUV peak.

\section{Discussion}

The conventional description of the star formation process begins with the (possibly triggered) gravitational collapse of massive gas clouds in the disk of a galaxy. These "super-clouds" may be in the form of $\mathrm{HI}$ or $\mathrm{H}_{2}$, depending on the physical conditions in the ISM (e.g. Elmegreen 1991 and references therein; Elmegreen 1993). The denser parts of these clouds cool further (and turn into $\mathrm{H}_{2}$ if they aren't already in that form) in giant molecular clouds (GMCs); stars then form and evolve in these GMCs (e.g. Shu et al. 1993). In this picture, a small fraction of the gas is consumed in the star formation process, and a larger fraction may be ionized and/or evacuated by stellar winds and supernovae. Some photodissociation of $\mathrm{H}_{2}$ into $\mathrm{HI}$ has also been observed on the surfaces of specific GMCs in our Galaxy (e.g. Blitz 1993, section 6; Andersson \& Wannier 1993; Kuchar \& Bania 1993) and column densities of order $1 \times 10^{21} \mathrm{~cm}^{-2}$ have been reported; but the photodissociation process is usually not thought of as being responsible for producing a substantial fraction of the Galactic H I. However, our model for the morphology of the UV, $\mathrm{H} \alpha$, and $\mathrm{HI}$ in M81 leads to the conclusion that a large fraction, and possibly all, of the $\mathrm{H}$ in the inner spiral arms of that galaxy is a photodissociation product.

\subsection{A preliminary model for the morphology}

The $\mathrm{H}$ I, $\mathrm{H} \alpha$, and FUV structures observed in M81 can be understood if "chimneys", like those proposed by Norman \& Ikeuchi (1989), are common in the spiral arms of this galaxy. In this picture, holes $\gtrsim 100$ pc in size are blown out of the galactic disk by con- 
centrations of high-mass star formation activity, similar to that discovered above the Galactic H II region IC1805 by Normandeau, Taylor, \& Dewdney (1996). From our vantage point above the disk of M81, such concentrations of star-forming activity are seen essentially free of obscuration, since the hot stars have evacuated or destroyed most of the intervening dust in M81. The gas in M81 is largely in the form of $\mathrm{H}_{2}$ at least in the main part of the disk; $\mathrm{H} \mathrm{I}$ is produced extensively by UV photons from the young stars impinging on the inner surfaces of these structures. The detailed structure of such an $\mathrm{H}$ I layer may resemble the H I morphology of the Galactic star-forming region G216-2.5, which has been explained as a PDR by Williams \& Maddalena (1996). This region shows a layer of $\mathrm{H} \mathrm{I} \approx 50 \mathrm{pc}$ thick spread over $300 \mathrm{pc}$ on the outer surface of a molecular cloud; the H I column density is of order $0.2 \times 10^{21} \mathrm{~cm}^{-2}$. The cause of the photodissociation is thought to be two young stars located $\sim 50 \mathrm{pc}$ from the $\mathrm{H}$ i layer.

In Fig. 8 we show a simplified cross-sectional sketch of the various morphologies which may arise in such a picture. The observer views the galaxy disk from above. Case I illustrates the IC1805 geometry, which forms the basis for our model. A young star cluster has formed just above the mid-plane in a dense layer of molecular gas. The $\mathrm{H}_{2}$ layer is shown in the sketch as a smooth medium but will have structure. If $\mathrm{O}$ stars are present, the accompanying $\mathrm{H} \alpha$ will be roughly coincident with the UV. Since the star-formation activity has opened a chimney up into the halo, the observer sees the optical and UV emission with little obscuration, even though the $\mathrm{H}_{2}$ layer underneath the bottom of the chimney will contain dust and may be opaque. The $\mathrm{HI}$, produced from the $\mathrm{H}_{2}$ by photodissociation, is spread over the inner surface of the chimney and will appear to the observer to be enhanced at the edges of the region, owing to the longer lines of sight there. Case II is the same geometry as Case I, but since the chimney has blown out on the far side of a more-or-less opaque disk of gas, the observer may measure nothing more than a patch of $\mathrm{H} \mathrm{I}$ emission.

Case III in Fig $\$$ shows a particularly energetic star-forming region located very close to the midplane of the galaxy. In this case, chimneys may blow out on both sides of the disk, leaving a hole right through the galaxy. The photodissociated $\mathrm{H}_{2}$ will appear to the observer as an H I "cocoon" wrapped around the boundary of the UV-emitting region, as seen e.g. in Field 3. Case IV is a one-sided version of Case III, formed in a non-uniform part of the $\mathrm{H}_{2}$ layer; it is a more energetic example of the model for G216-2.5 by Williams \& Maddelena (1996).

\subsection{Production of $\mathbf{H}_{\mathrm{I}}$ by photodissociation}

A simple way to obtain a rough order of magnitude for the $\mathrm{HI}$ column density in a PDR is to consider the case where the dust opacity controls the depth to which UV penetrates the surfaces of molecular clouds. This will occur when self-shielding by $\mathrm{H}_{2}$ is relatively unimportant (low volume density). In this case, the UV penetrates and dissociates to a depth where the UV opacity $\tau_{\mathrm{UV}} \approx 1$. With the usual values for dust-to-gas ratio appropriate for translucent lines of sight in the Galaxy, namely, $\mathrm{N}(\mathrm{H})=1.8 \times 10^{21} \times$ $\mathrm{A}_{\mathrm{V}}$ atoms $\mathrm{cm}^{-2}$ (Bohlin, Savage, \& Drake 1978), and using $\mathrm{A}_{\mathrm{FUV}}=2.54 \times \mathrm{A}_{\mathrm{V}}$ (Seaton 1979), we have $\mathrm{A}_{\mathrm{FUV}}=1$ at $\mathrm{N}(\mathrm{H})=8 \times 10^{20}$ atoms $\mathrm{cm}^{-2}$, predominantly in the form of $\mathrm{H}$ IThis value is close to the threshold values of $\mathrm{N}(\mathrm{HI})$ found in the immediate neighborhood of FUV concentrations in the five fields under discussion in this paper and shows that the dissociation picture provides the right order of magnitude for the H c column density.

\subsubsection{A quantitative model:}

Many authors have developed detailed models of PDRs including self-shielding; the review by Hollenbach \& Tielens (1995) lists many relevant references. In particular, H I production in PDRs has been considered by Federman, Glassgold, \& Kwan (1979) for equilibrium models; time-dependent models have been computed by Roger and Dewdney (1992) and by Bertoldi \& Draine (1996). Sternberg (1988) has provided a convenient analytic solution to a simple semi-infinite slab geometry in equilibrium with UV radiation incident on one side. The solution gives the steady-state H I column density along a line of sight perpendicular to the face of the slab, as a function of the FUV flux $\chi$ in units of Draine's (1978) standard values $\chi_{0}$ for the ISM near the sun, and the total volume density of $\mathrm{H}$ nucleii $n=n(\mathrm{HI})+2 n\left(\mathrm{H}_{2}\right)$ in the gas. Using the parameters in this equation adopted by Madden et al. (1993) we have:

$$
\mathrm{N}(\mathrm{H} \mathrm{I})=5 \times 10^{20} \times \ln \left[1+\left(\chi / \chi_{0}\right)(90 / n)\right]
$$

As a specific example, we apply this model to the $\mathrm{HI}$ and FUV associated with the HII region at 
$9^{\mathrm{h}} 50^{\mathrm{m}} 44^{\mathrm{s}}, 69^{\circ} 17^{\prime} 30^{\prime \prime}$ (Field 3), which is the region described earlier where the $\mathrm{HI}$ appears to surround the UV and $\mathrm{H}$ II concentrations. The bright $\mathrm{H}$ II region associated with the northern UV peak is \#44 in the catalog of Petit et al. The UV flux of this region integrated over a circular aperture $16^{\prime \prime}$ in diameter on the full-resolution image is given by Hill et al. as $\mathrm{f}_{150}$ $=1.39 \times 10^{-15} \mathrm{erg} \mathrm{cm}^{-2} \mathrm{~s}^{-1} \AA^{-1}$; we obtain a somewhat larger value of $2.55 \times 10^{-15}$ by direct integration of the contours of our (smoothed) image in Fig. 5 over the same area.

This observed flux must be corrected for extinction at $150 \mathrm{~nm}$. There are two parts to this correction: Galactic foreground extinction, and extinction in M81 itself. Consistent with our conclusion that the UV and H II regions are essentially "naked", the extinction in M81 itself is taken to be completely "gray", caused by similar obscuration of parts of the emitting region both at UV and $\mathrm{H} \alpha$. This kind of extinction can be revealed by a comparison of the thermal radio flux density of an $\mathrm{H}$ II region with its $\mathrm{H} \alpha$ flux. With this method, Kaufman et al. (1987) derive an average extinction (at $\approx 650 \mathrm{~nm}$ ) for $\mathrm{H} \mathrm{II}$ regions in $\mathrm{M} 81$ of $\mathrm{A}_{\alpha} \approx 0.86 \mathrm{mag}$, of which they estimate $\approx 0.23$ mag is Galactic foreground extinction. In our model, this foreground extinction is the only part which will increase at shorter wavelengths. Taking $\mathrm{A}_{150}=3.1 \times \mathrm{A}_{\alpha}$ (Seaton 1979), we estimate that for $\mathrm{H}$ II region \#44, $\mathrm{A}_{150}=3.1 \times 0.23+0.63 \approx 1.34 \mathrm{mag}$. The corrected FUV flux at the earth is then $\mathrm{f}_{150}^{\prime}=$ $8.77 \times 10^{-15} \mathrm{erg} \mathrm{cm}^{-2} \mathrm{~s}^{-1} \AA^{-1}$, corresponding to the UV flux from $\sim 9$ stars of type $\mathrm{O} 9 \mathrm{~V}$ at a distance to M81 of 3.7 Mpc. Normandeau et al. (1996) list 9 O stars as the major power sources in the Galactic region IC1805, which is our model for the "chimneys" in M81.

The following step is to compute the UV flux at the location of the H I "blanket". The distance between the dissociating stars and the $\mathrm{H}$ I near region \#44 in M81 is about 1/3 of our resolution FWHM, or about $50 \mathrm{pc}$, which is also similar to the geometry in IC1805 and the same as the value estimated by Williams \& Maddalena for G216-2.5. The flux ratio is therefore $\left(3.7 \times 10^{6} / 50\right)^{2}=5.5 \times 10^{9}$ for a distance to M81 of $3.7 \mathrm{Mpc}$, so the FUV flux incident on the molecular cloud is $\chi=8.77 \times 10^{-15} \times 5.5 \times 10^{9}$ $=4.8 \times 10^{-5} \mathrm{erg} \mathrm{cm}^{-2} \mathrm{~s}^{-1} \AA^{-1}$. The value for $\chi_{0}$ at $150 \mathrm{~nm}$ can be read from Fig.1 in Van Dishoeck \& Black (1988) as $2 \times 10^{5}$ photons $\mathrm{cm}^{-2} \mathrm{~s}^{-1} \AA^{-1} \times$ $1.32 \times 10^{-11} \mathrm{ergs} /$ photon $=$
$2.64 \times 10^{-6} \mathrm{erg} \mathrm{cm}^{-2} \mathrm{~s}^{-1} \AA^{-1}$ so that the ratio $\chi / \chi_{0} \approx$ 18.

From equation 1 the density $n$ of the underlying gas can be estimated from the observed FUV flux and the H I column density of the PDR. The extended regions of $\mathrm{HI}$ emission in close proximity to $\mathrm{H}$ II region \#44 have $\mathrm{N}(\mathrm{HI})=2.5 \times 10^{21} \mathrm{~cm}^{-2}$. There are more localised areas reaching a brightness of two or three times this value, but in our picture these enhancements are the result of viewing a thick blanket of $\mathrm{H} \mathrm{I}$ more or less edge-on (cf. Fig.8). Taking G216-2.5 again as a model, the line of sight may reach $300 \mathrm{pc}$ tangential to a layer of intrinsic thickness of only 50 pc. Accounting for our $150 \mathrm{pc}$ beam, the net enhancement would be of order $(300 / 50) \times(50 / 150)=2$, in rough agreement with our $\mathrm{H}$ I observations. Using $\mathrm{N}(\mathrm{H} \mathrm{I})=2.5 \times 10^{21} \mathrm{~cm}^{-2}$ and $\chi / \chi_{0}=18$ in equation 1, we compute the density of the underlying gas to be $n \approx 10 \mathrm{~cm}^{-3}$. Although the accuracy of this result is not very high, the value is about the same as that used by Rand et al. (1992) in their "best" photodissociation models for the H I in M51. For G216-2.5, we calculate from the values in Williams \& Maddelena that $n(\mathrm{H} \mathrm{I}) \approx \mathrm{N}(\mathrm{HI}) / \mathrm{L}=1.5 \mathrm{~cm}^{-3}$ with $\mathrm{L}=50 \mathrm{pc}$, significantly less than the value $n=n(\mathrm{HI})+2 n\left(\mathrm{H}_{2}\right)$ $=10 \mathrm{~cm}^{-3}$ derived heref. However, the detected $\mathrm{HI}$ will have been heated by the photodissociation process to $\sim 80 \mathrm{~K}$, and the factor of $\sim 6$ in density is easily understood if the kinetic temperature of the precursor $\mathrm{H}_{2}$ is less than a plausible $\sim 15 \mathrm{~K}$.

\subsubsection{The molecular content of M81:}

The photodissociation picture provides a reasonable explanation for the $\mathrm{H}$ I morphologies and column densities in the spiral arms of M81. The underlying gas is of modest density, $\sim 10 \mathrm{H}$ nucleii $\mathrm{cm}^{-3}$, but must be widespread, since every UV source has photodissociated $\mathrm{HI}$ in its immediate vicinity. The amount of $\mathrm{H}_{2}$ present in the spiral arms of M81 is uncertain, but it is likely to be at least as much as the $\mathrm{HI}$, and may be significantly greater. This result contrasts with the $\mathrm{CO}(1-0)$ surveys of M81. For instance, using the usual factor of Bloemen et al. (1986) to convert $\mathrm{I}_{\mathrm{CO}}$ to $\mathrm{N}\left(\mathrm{H}_{2}\right)$, Brouillet et al. (1991) conclude that the $\mathrm{H}_{2}$ mass in the annular region $4 \leq \mathrm{R} \leq 7$ kpc containing the major spiral arms in M81 is only $20 \%$ of the Hi mass in the same area. The ratio

\footnotetext{
${ }^{5}$ The gas in the H I zone of the PDR will be mostly in the form of $\mathrm{HI}$.
} 
varies from $10 \%$ to $40 \%$ in specific regions along the spiral arms as observed with the $12 \mathrm{~m}$ NRAO millimeter radio telescope (beam $\mathrm{FWHM} \approx 1^{\prime}$ ) when the $\mathrm{H} \mathrm{I}$ is smoothed to the same resolution (Brouillet et al. Table 3). The conventional conversion factor linking ${ }^{12} \mathrm{CO}(1-0)$ surface brightness and $\mathrm{H}_{2}$ column density must underestimate the quantity of molecular gas in the spiral arms of M81 by at least a factor of five. A similar discrepancy was noted by Kaufman et al. (1989b) in their study of the dust lanes in M81.

Allen (1996) reviewed the case for using ${ }^{12} \mathrm{CO}(1-$ 0 ) brightnesses to infer $\mathrm{H}_{2}$ column densities in galaxy disks and concluded that this practice is unreliable. Our present result in M81 contributed to that conclusion.

\section{Conclusions}

Our detailed morphological study of the FUV, $\mathrm{H} \alpha$, and $\mathrm{H} \mathrm{I}$ in $\mathrm{M} 81$ at a resolution of $150 \mathrm{pc}$ indicates that most of the $\mathrm{HI}$ is $\mathrm{UV}$-dissociated $\mathrm{H}_{2}$, at least in the inner spiral arms of this galaxy. In this picture, the $\mathrm{H} \mathrm{I}$ in the spiral arms is a product of the star formation process rather than a precursor.

A corollary of this picture is that the disk of M81 must harbor a large reservoir of molecular gas which has so far escaped detection, e.g. in the ${ }^{12} \mathrm{CO}(1-0)$ line. Since M81 has a generally low level of heat production, as evidenced by the faintness of the nonthermal radio continuum and the generally low surface brightness in $\mathrm{H} \alpha$ emission, this molecular gas is likely to be generally cold and therefore faint in e.g. ${ }^{12} \mathrm{CO}(1-0)$ emission. The "skins" of these molecular clouds may be sufficiently warmed by nearby UV sources to be visible in ${ }^{12} \mathrm{CO}(1-0)$, but this emission will have a small spatial filling factor. The underlying cold clouds may be visible in the inner disk of M81 as broad emission lines of ${ }^{12} \mathrm{CO}(1-0)$ characteristic of GMCs, but unusually faint and with abnormally low values of the ${ }^{12} \mathrm{CO}(2-1) /{ }^{12} \mathrm{CO}(1-0)$ ratio. Such molecular clouds have been found in the inner disk of M31 by Allen \& Lequeux (1993) and by Loinard et al. (1995).

We thank D. Westpfahl, M. Kaufman and N. Devereux for providing the data for this study, and for helpful discussions on its use and interpretation. We are also grateful to A. Poglitsch for introducing the notion that PDRs may be ubiquitous in galaxy disks and to D. Hollenbach for suggesting relevant refer- ences on PDR models. It is a pleasure to acknowledge Colin Norman and all our colleagues at STScI and JHU for stimulating discussions. Laurent Loinard assisted with a critical reading of the manuscript and provided the drawing for Figure 8. JHK thanks the Director of STScI for supporting several visits to the Institute while this paper was being written, and the FCAR Québec Action Concertée for financial assistance. 


\section{REFERENCES}

Adler, D.A., \& Westpfahl, D.J. 1996, AJ, 111, 735

Allen, R.J. 1996, "Molecular Gas in Spiral Galaxies", in "New Extragalactic Perspectives in the New South Africa", ed. D.L. Block \& J.M. Greenberg (Kluwer; Dordrecht), 50

Allen, R.J., Atherton, P.D., \& Tilanus, R.P.J. 1985, in "Birth and Evolution of Massive Stars and Stellar Groups," ed. W. Boland \& H. Van Woerden (Reidel; Dordrecht), 243

Allen, R.J., Atherton, P.D., \& Tilanus, R.P.J. 1986, Nature, 319, 296

Allen, R.J., \& Lequeux, J. 1993, ApJ, 410, L15

Andersson, B.-G., \& Wannier, P. 1993, ApJ 402, 585

Bertoldi, F., \& Draine, B.T. 1996, ApJ, 458, 222

Blitz, L. 1993, in "Protostars \& Planets III", ed. E.H. Levy \& J.I. Lunine (Univ. Arizona Press; Tucson), 125

Bloemen, J.B., Strong, A.W., Blitz, L., Cohen, R.S., Dame, T.M., Grabelsky, D.A., Hermsen, W., Lebrun, F., Meyer-Hasselwander, H.A., \& Thaddeus, P. 1986, A\&A 154, 25

Bohlin, R.C., Savage, B.D., \& Drake, J.F. 1978, ApJ, 224, 132

Brouillet, N, Baudry, A., Combes, F., Kaufman, M., \& Bash, F. 1991, A\&A, 242, 35

De Vaucouleurs, G., de Vaucouleurs, A., Corwin, H.G., Buta, R.J., Paturel, G., \& Fouqué, P. 1991, "Third Reference Catalogue of Bright Galaxies" (Springer, New York)

Devereux, N.A., Jacoby, G., \& Ciardullo, R. 1995, AJ, 110, 1115

Draine, B.T. 1978, ApJS, 36, 595

Elmegreen, B.G. 1991, in "The Physics of Star Formation and Early Stellar Evolution", ed. C. Lada \& N. Kylafis (Kluwer), 35

Elmegreen, B.G. 1993, in "Protostars and Planets III", ed. E.H. Levy \& J.I. Lunine (Univ. Arizona Press; Tucson), 97

Federman, S.R., Glassgold, A.E., \& Kwan, J. 1979, ApJ, 227, 466

Hill, J.K. et al. 1995, ApJ, 438, 181

Hollenbach, D.J., \& Tielens, A.G.G.M. 1995, in "The Physics and Chemistry of Interstellar Molecular Clouds", ed. G. Winnewisser \& G.C. Pelz (Springer-Verlag; Berlin), 164

Israel, F.P., Goss., W.M., \& Allen, R.J. 1975, A\&A, 40, 421

Kaufman, M., Bash, F.N., Kennicutt, R.C.Jr., \& Hodge, P.W. 1987, ApJ, 319, 61

Kaufman, M., Bash, F.N., Hine, B., Rots, A.H., Elmegreen, D.M., Hodge, P.W. 1989a, ApJ, 345, 674

Kaufman, M., Elmegreen, D.M., \& Bash, F.N. 1989b, ApJ, 345, 697

Knapen, J.H., Beckman, J.E., Cepa, J., van der Hulst, J.M. \& Rand, R.J. 1992, ApJ, 385, L37

Knapen, J.H., \& Beckman, J.E. 1994, in "Physics of Gaseous and Stellar Discs of Galaxies", ed. I. King (A.S.P. Conference Series Vol. 66), 329

Knapen, J.H., \& Beckman, J.E. 1996, MNRAS, 283, 251

Kuchar, T.A., \& Bania, T.M. 1993, ApJ, 414, 664

Loinard, L., Allen, R.J., \& Lequeux, J. 1995, A\&A, 301, 68

Madden, S.C., Geis, N., Genzel, R., Herrmann, F., Jackson, J., Poglitsch, A., Stacey, G.J., \& Townes, C.H. 1993, ApJ, 407, 579

Norman, C.A., Ikeuchi, S. 1989, ApJ, 345, 372 
Normandeau, M., Taylor, A.R., \& Dewdney, P.E. 1996, Nature, 380, 687

Petit, H., Sivan, J.-P., \& Karachentsev, I.D. 1988, A\&AS, 74, 475

Rand, R.J., Kulkarni, S.R., \& Rice, W. 1992, ApJ, 390, 66

Roger, R.S., \& Dewdney, P.E. 1992, ApJ, 385, 536

Seaton, M.J. 1979, MNRAS, 187, 73p

Shaya, E.J., \& Federman, S.R. 1987, ApJ, 319, 76

Shu, F., Najita, J., Galli, D., Ostriker, E., Lizano, S. 1993, in "Protostars and Planets III", ed. E.H. Levy \& J.I. Lunine (Univ. Arizona Press; Tucson), 3

Stacey, G.J., Geis, N., Genzel, R., Lugten, J.B., Poglitsch, A., Sternberg, A., \& Townes, C.H. 1991, ApJ, 373, 423

Stecher, T.P., \& Williams, D.A. 1967, ApJ, 149, L29

Stecher, T.P. et al. 1992, ApJ, 395, L1

Sternberg, A. 1988, ApJ, 332, 400

Tilanus, R.P.J., \& Allen, R.J. 1987, in "Star Formation in Galaxies", ed. C.J. Lonsdale Persson (NASA CP-2466), 309

Tilanus, R.P.J., \& Allen, R.J. 1989, ApJ, 339, L57

Tilanus, R.P.J. \& Allen, R.J. 1990, in "The Interstellar Medium in External Galaxies: Summaries of Contributed Papers," ed. D.J. Hollenbach and H.A. Thronson, Jr. (NASA Conference Publication 3084), 298

Tilanus, R.P.J., \& Allen, R.J. 1991, A\&A, 244, 8

Tilanus, R.P.J., \& Allen, R.J. 1993, A\&A, 274, 707

Tilanus, R.P.J., Allen, R.J., van der Hulst, J.M., Crane, P.C., \& Kennicutt, R.C. 1988, ApJ, 330, 667

Van der Kruit, P.C., Allen, R.J., \& Rots, A.H. 1977, A\&A, 55, 421

Van Dishoeck, E.F., \& Black, J.H. 1988, ApJ, 334, 771

Vogel, S.N., Kulkarni, S.R., \& Scoville, N.Z. 1988, Nature, 334, 402

Williams, J.P., \& Maddelena, R.J. 1996, ApJ, 464, 247

This 2-column preprint was prepared with the AAS LATEX macros v4.0. 
Fig. 1.- Contour representation of the far-UV (FUV) image of M81 taken with the Ultraviolet Imaging Telescope on the ASTRO-1 Mission, overlaid on a grey-scale representation of the VLA HI map of M81. The numbered squares indicate five sub-fields, discussed in detail in the text. The resolution of both images is $9^{\prime \prime}=150 \mathrm{pc}$. Contour levels for the FUV are 2.3, 3.1, 4.6, 6.2, 7.7, 9.2, 13.9, 18.5 and $36.9 \times 10^{-18} \mathrm{erg} \mathrm{cm}^{-2} \mathrm{~s}^{-1} \AA^{-1} \mathrm{arcsec}^{-2}$. Grey scales for the HI vary linearly from 1.5 (light) to $7.3 \times 10^{21}$ atoms $\mathrm{cm}^{-2}$ (dark).

Fig. 2.- Radial emission profiles of H I (bottom panel), FUV (middle panel), and $\mathrm{H} \alpha$ (top panel) in the disk of M81 on logarithmic scales. Units are $\log$ of $\mathrm{N}(\mathrm{HI})$ in atoms $\mathrm{cm}^{-2}, \log$ of FUV surface brightness in $\operatorname{erg~cm}{ }^{-2} \mathrm{~s}^{-1} \AA^{-1} \operatorname{arcsec}^{-2}$, and log of $\mathrm{H} \alpha$ surface brightness in $\mathrm{erg} \mathrm{cm}^{-2} \mathrm{~s}^{-1} \operatorname{arcsec}^{-2}$.

Fig. 3.- Field 1 in M81, as indicated in Fig.1 1 and discussed in the text. Contours in all three panels are of the FUV emission, contour levels as in Fig.1. The contours are overlaid on grey-scale representations of the FUV (left panel, grey-scales from 0.8 [light] to $15 \times 10^{-18} \mathrm{erg} \mathrm{cm}^{-2} \mathrm{~s}^{-1} \AA^{-1} \operatorname{arcsec}^{-2}$ [dark]); H $\alpha$ (middle panel; greys from 0.5 to $95 \times 10^{-17} \mathrm{erg} \mathrm{cm}^{-2} \mathrm{~s}^{-1} \operatorname{arcsec}^{-2}$; and HI (right panel, grey levels as in Fig. (1). Positions of the dust filaments as measured by Kaufman, Elmegreen \& Bash (1989) are indicated by the line segments drawn in the middle panel.

Fig. 4.- As Fig.3, now for Field 2.

Fig. 5.- As Fig.3, now for Field 3.

Fig. 6.- As Fig.3, now for Field 4.

Fig. 7.- As Fig.3, now for Field 5.

Fig. 8.- Cartoon sketch of how various "Norman-Ikeuchi" chimneys could occur in the disk of a galaxy (Norman \& Ikeuchi 1989, see their Figs. 4 \& 5). The transverse size scale of a large chimney (powered by a cluster of O stars) may reach a few hundred pc. In a dense layer of $\mathrm{H}_{2}$, these structures develop envelopes of $\mathrm{H}$ I which partially surround the star-forming region. When viewed from a distant vantage point, such structures may explain the morphology of the UV, $\mathrm{HI}$, and $\mathrm{H} \alpha$ which we observe in M81. 


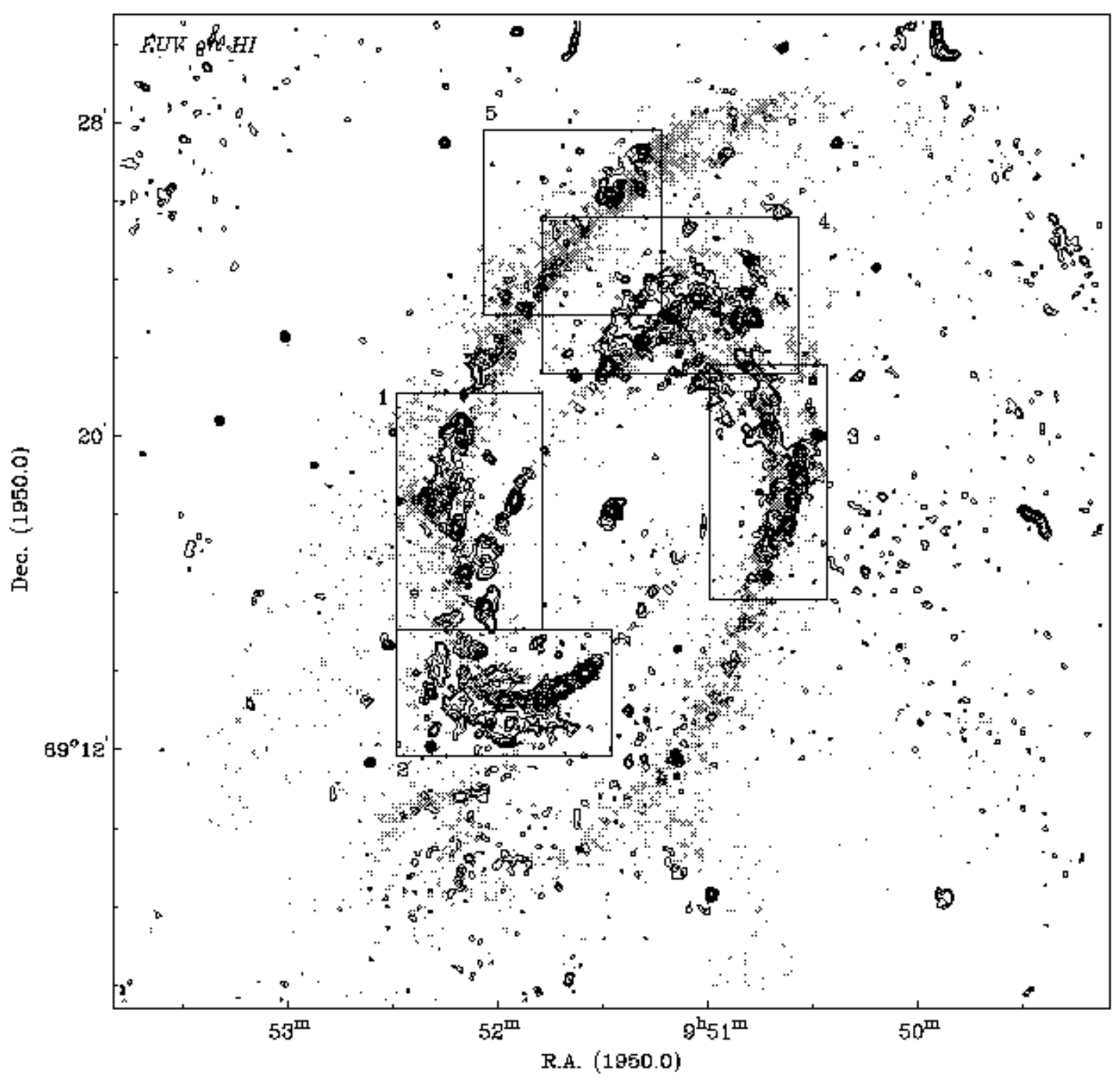




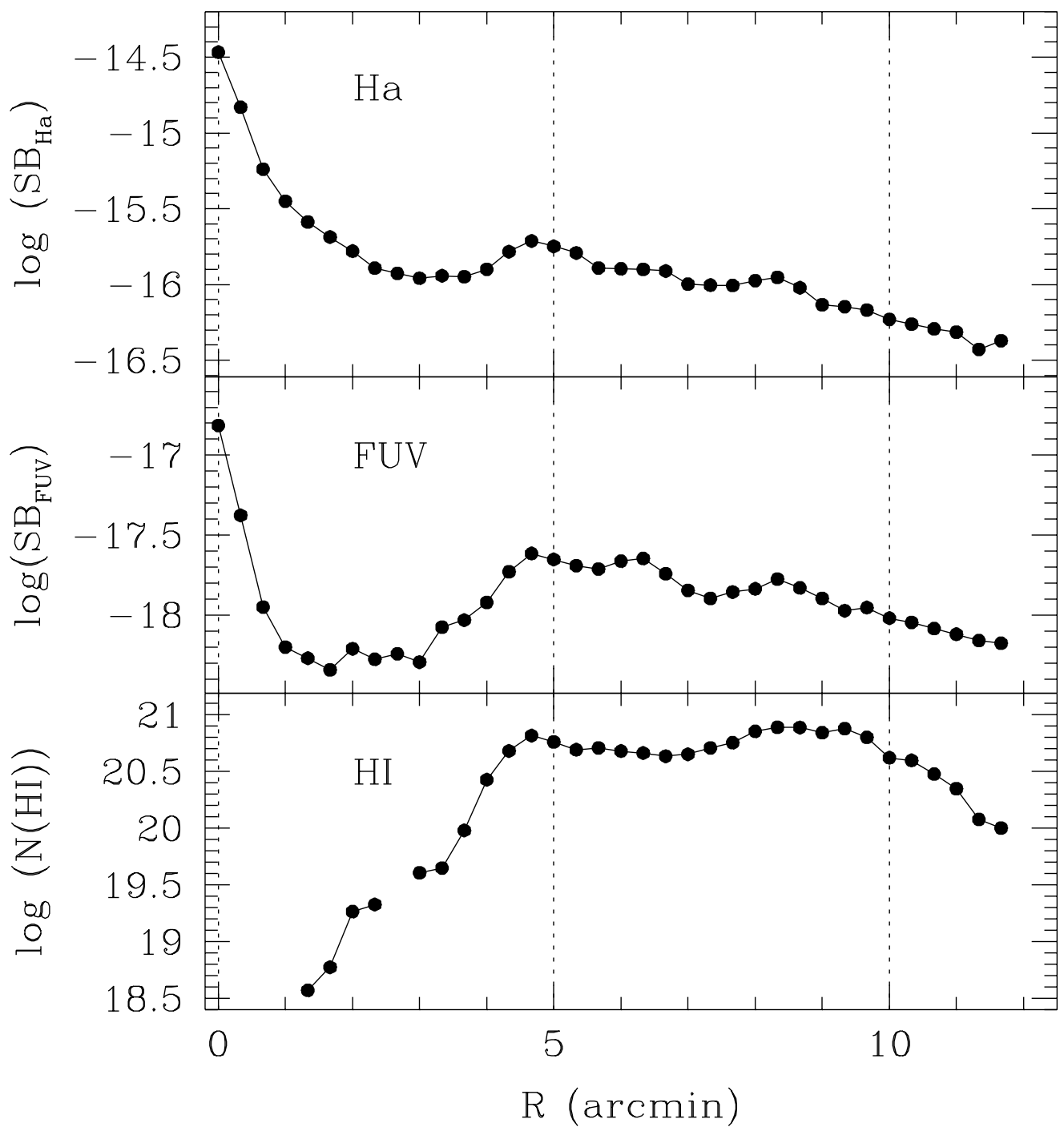




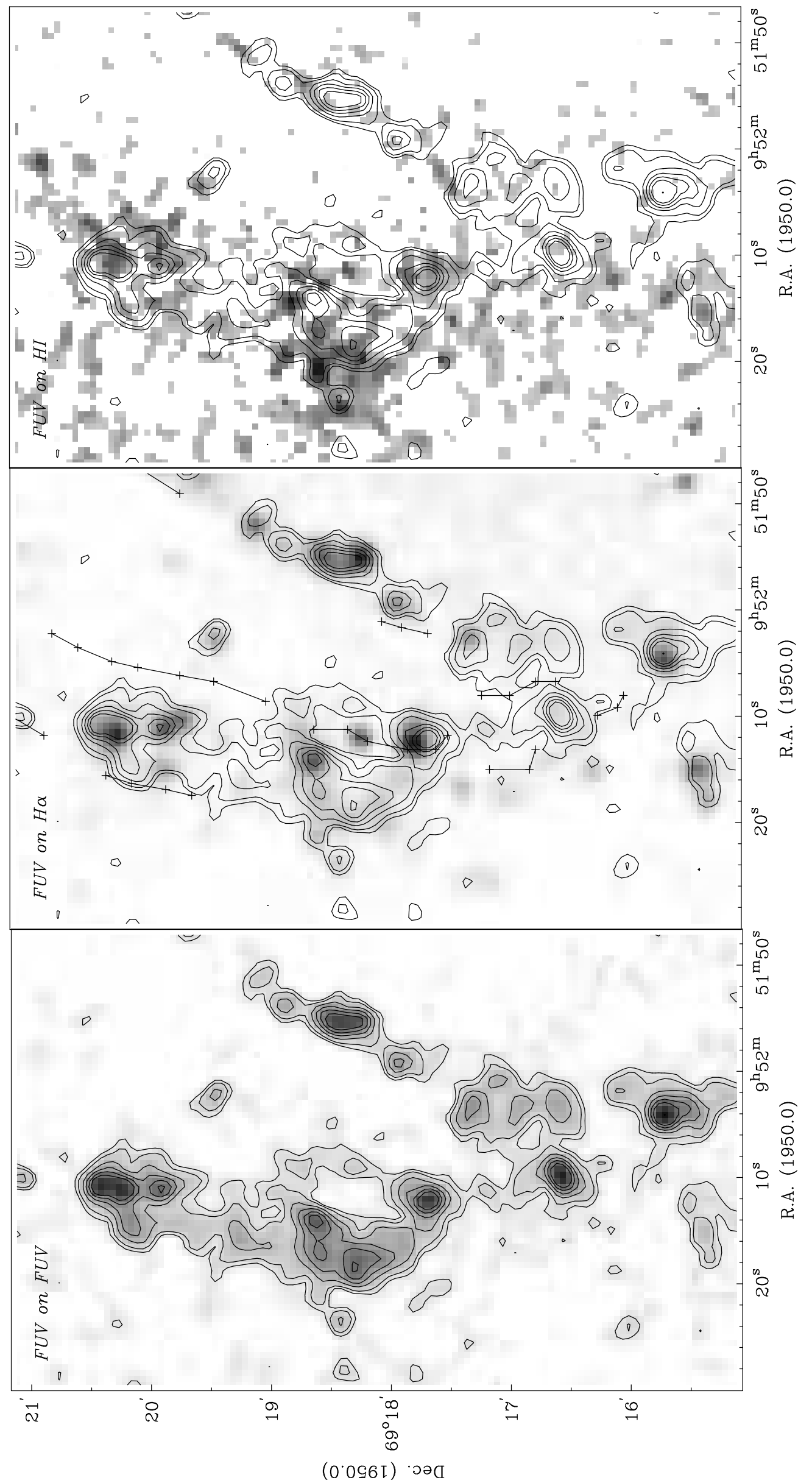




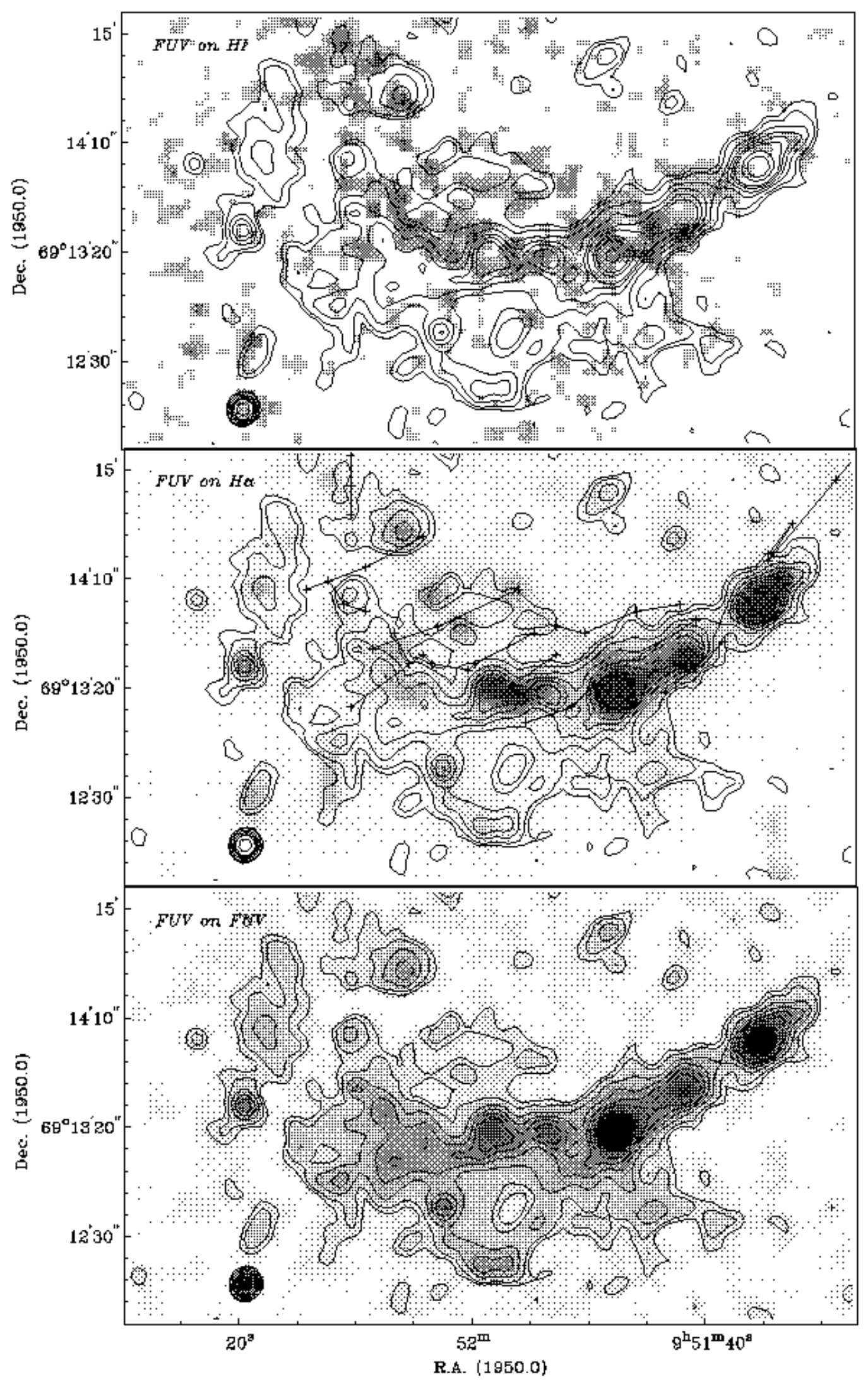




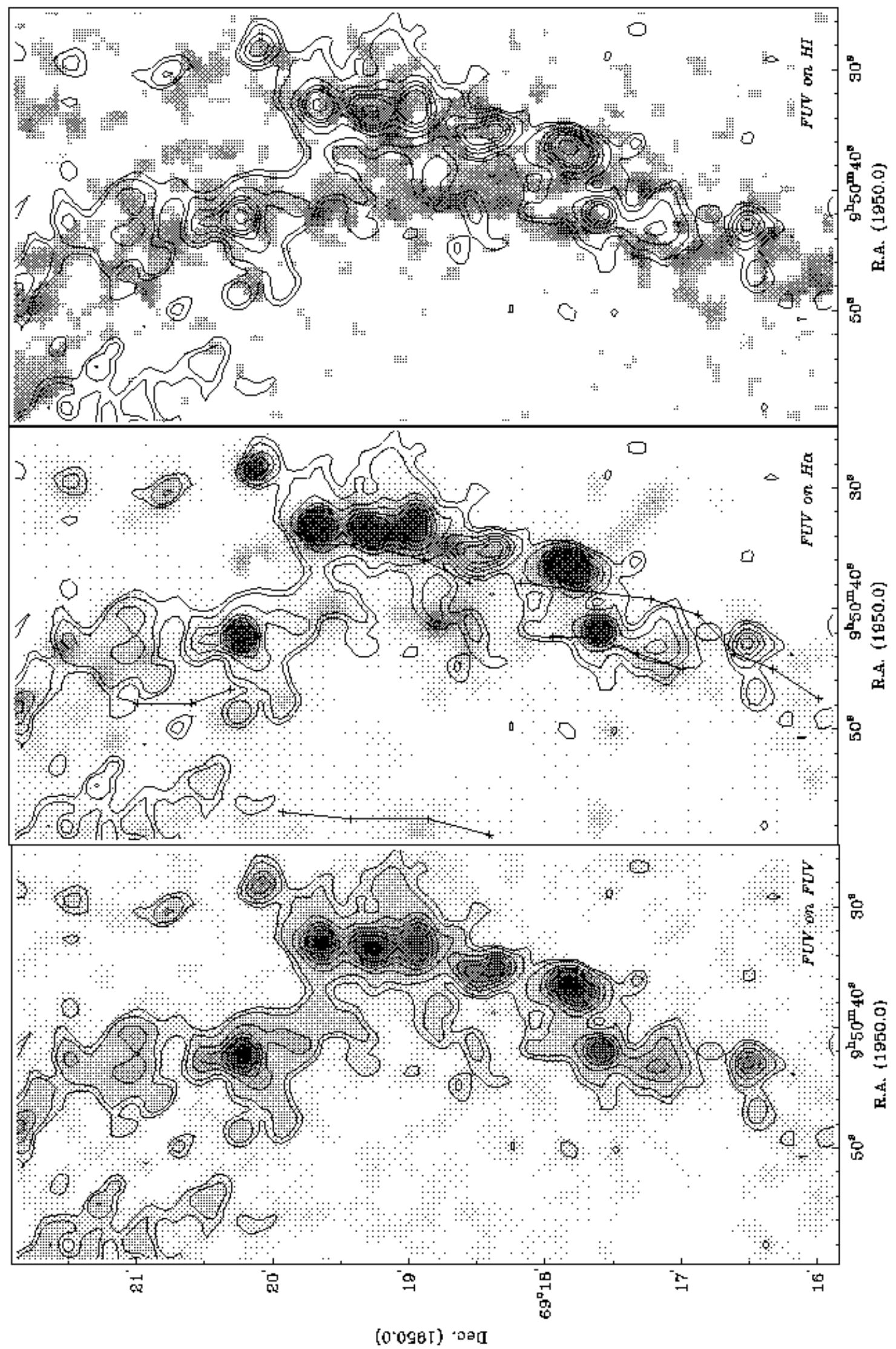




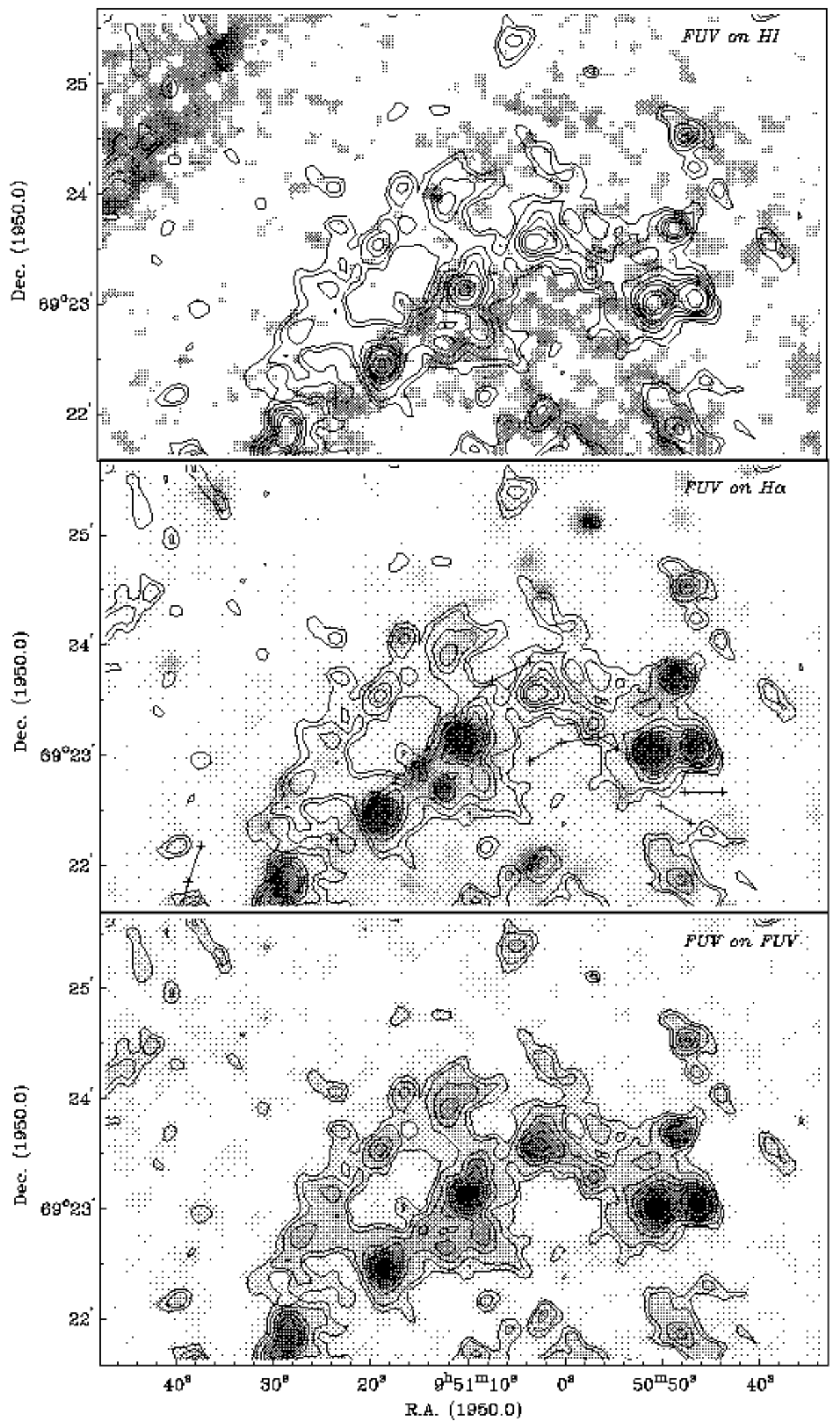




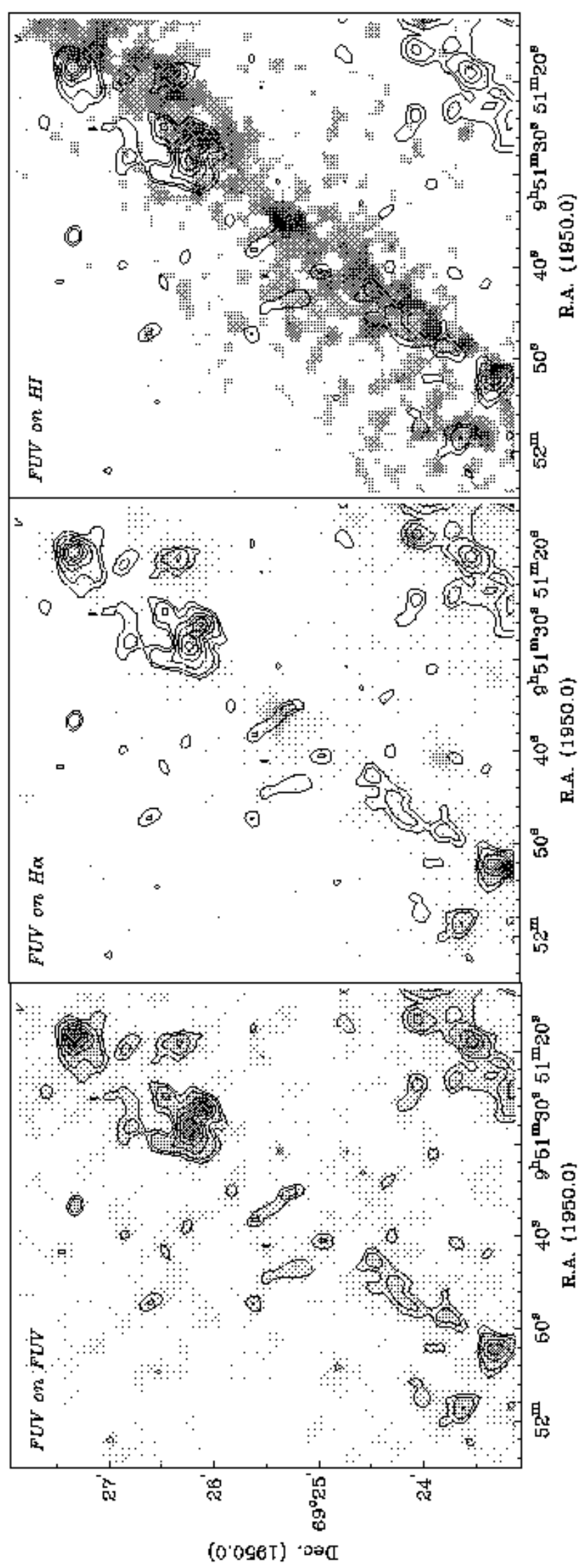



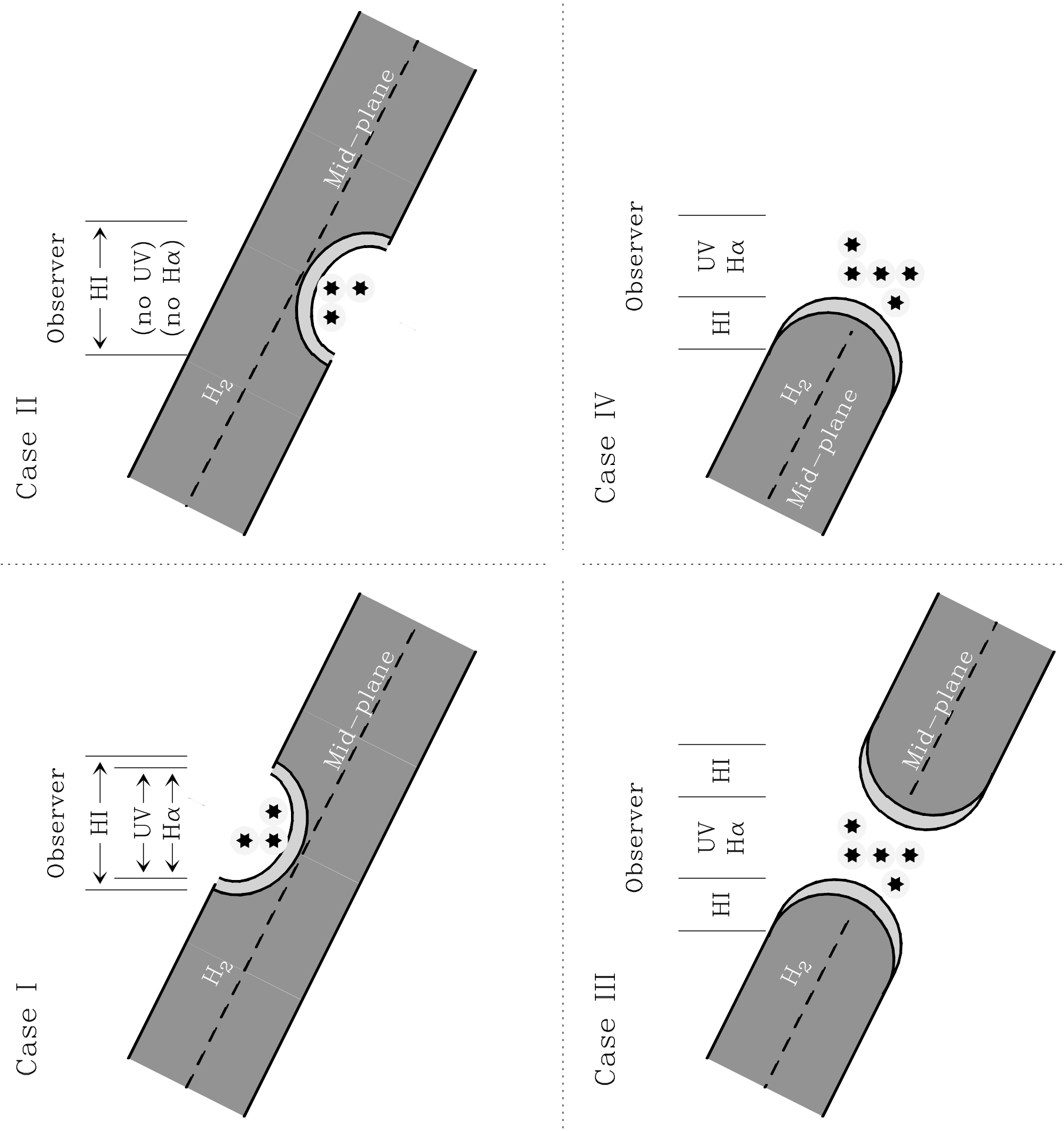